\title{
Monitoring metabolism of synthetic cannabinoid 4F-MDMB-BINACA via high-resolution mass spectrometry assessed in cultured hepatoma cell line, fungus, liver microsomes and confirmed using urine samples
}

\author{
Huey Sze Leong ${ }^{1,2} \mathbb{D} \cdot$ Shimpei Watanabe ${ }^{3,4,7} \cdot$ Unnikrishnan Kuzhiumparambil ${ }^{5} \cdot$ Ching Yee Fong $^{6} \cdot \mathrm{Hooi} \mathrm{Yan} \mathrm{Moy}^{6}$. \\ Yi Ju Yao ${ }^{6}$ Paul K. Witting ${ }^{2}$. Shanlin Fu ${ }^{1}$
}

Received: 8 October 2020 / Accepted: 3 December 2020 / Published online: 29 December 2020

(c) The Author(s) 2020

\begin{abstract}
Purpose A tert-leucinate derivative synthetic cannabinoid, methyl (2S)-2-([1-(4-fluorobutyl)-1H-indazole-3-carbonyl] amino)-3,3-dimethylbutanoate (4F-MDMB-BINACA, 4F-MDMB-BUTINACA or 4F-ADB) is known to adversely impact health. This study aimed to evaluate the suitability of three different modes of monitoring metabolism: HepG2 liver cells, fungus Cunninghamella elegans (C. elegans) and pooled human liver microsomes (HLM) for comparison with human invivo metabolism in identifying suitable urinary marker(s) for 4F-MDMB-BINACA intake.

Methods Tentative structure elucidation of in-vitro metabolites was performed on HepG2, C. elegans and HLM using liquid chromatography-tandem mass spectrometry and high-resolution mass spectrometry analysis. In-vivo metabolites obtained from twenty authentic human urine samples were analysed using liquid chromatography-Orbitrap mass spectrometry.

Results Incubation with HepG2, C. elegans and HLM yielded nine, twenty-three and seventeen metabolites of 4F-MDMBBINACA, respectively, formed via ester hydrolysis, hydroxylation, carboxylation, dehydrogenation, oxidative defluorination, carbonylation or reaction combinations. Phase II metabolites of glucosidation and sulfation were also exclusively identified using $C$. elegans model. Eight in-vivo metabolites tentatively identified were mainly products of ester hydrolysis with or without additional dehydrogenation, $N$-dealkylation, monohydroxylation and oxidative defluorination with further oxidation to butanoic acid. Metabolites with intact terminal methyl ester moiety, i.e., oxidative defluorination with further oxidation to butanoic acid, were also tentatively identified.

Conclusions The in-vitro models presented proved useful in the exhaustive metabolism studies. Despite limitations, HepG2 identified the major 4F-MDMB-BINACA ester hydrolysis metabolite, and C. elegans demonstrated the capacity to produce a wide variety of metabolites. Both $C$. elegans and HLM produced all the in-vivo metabolites. Ester hydrolysis and ester hydrolysis plus dehydrogenation 4F-MDMB-BINACA metabolites were recommended as urinary markers for 4F-MDMBBINACA intake.
\end{abstract}

Keywords 4F-MDMB-BINACA metabolism - 4F-MDMB-BUTINACA (4F-ADB) · HepG2 · Cunninghamella elegans · Human liver microsomes · Urinary marker of 4F-MDMB-BINACA intake

Shanlin Fu

Shanlin.Fu@uts.edu.au

1 Centre for Forensic Science, School of Mathematical and Physical Sciences, University of Technology Sydney, PO Box 123, Broadway, NSW 2007, Australia

2 Discipline of Pathology, Faculty of Medicine and Health, The Charles Perkins Centre, The University of Sydney, Johns Hopkins Drive, Camperdown, NSW 2006, Australia

3 Division of Drug Research, Department of Biomedical and Clinical Sciences, Linköping University, 58183 Linköping, Sweden
4 Deparment of Forensic Genetics and Forensic Toxicology, National Board of Forensic Medicine, Artillerigatan 12, 58758 Linköping, Sweden

5 Climate Change Cluster, University of Technology Sydney, PO Box 123, Broadway, NSW 2007, Australia

6 Analytical Toxicology Laboratory, Applied Sciences Group, Health Sciences Authority, 11 Outram Road, Singapore 169078, Singapore

7 Present Address: Forensic Science Group, Photon Science Research Division, RIKEN, SPring-8 Center, Hyogo 679-5148, Japan 


\section{Introduction}

Synthetic cannabinoids (SCBs) are agonists at cannabinoid receptor type $1\left(\mathrm{CB}_{1}\right)$ and type $2\left(\mathrm{CB}_{2}\right)$, where they elicit their main effects. Due to their similar physiological effects to the principal psychoactive component of cannabis, $\Delta^{9}$-tetrahydrocannabinol (THC), SCBs are gaining popularity and are often abused as recreational drugs. However, most of the SCBs are full agonists at $\mathrm{CB}_{1}$ and $\mathrm{CB}_{2}$ receptors, having a higher risk of undesirable side effects when compared to THC which is a partial agonist [1]. Methyl (2S)-2-([1-(4-fluorobutyl)- $1 H$-indazole3-carbonyl]amino)-3,3-dimethylbutanoate (4F-MDMBBINACA, 4F-MDMB-BUTINACA or 4F-ADB), found in numerous $\mathrm{SCB}$ product seizures, has been reported by various law enforcement since 2018 [2]. The tert-leucine derived indazole-3-carboxamide analog 4F-MDMBBINACA postulated to be a potent agonist of the $\mathrm{CB}_{1}$ receptor based upon its structural similarity to methyl2-([1-(5-fluoropentyl)- $1 H$-indazole-3-carbonyl $]$ amino)3,3-dimethylbutanoate (5F-MDMB-PINACA) [3, 4], has significant abuse potential and causes central nervous system depression. Death involving these drugs have been reported [5-9], and this raises public health and social concerns. Moreover, 4F-MDMB-BINACA has been listed in Schedule II of the Convention on Psychotropic Substances of 1971 recently.

The proliferation of SCBs has become a global challenge as new compounds are rapidly introduced into the illegal drug market to evade existing drug laws. Identifying SCB intake and its correlating specific adverse effects require rapid elucidation of these SCBs. Since most SCBs are found extensively in metabolized forms in urine, the identification of metabolites is of vital importance for forensic and clinical toxicologists. Furthermore, some hydroxylated urinary metabolites have been found to be active and more toxic than the parent SCBs themselves, emphasizing the importance of metabolism study in identifying potential toxic metabolites $[10,11]$.

Traditional in-vivo metabolism studies to generate human metabolites of drugs relied heavily on the use of whole animal model systems, which are expensive, limited by drug administration amount, influenced by species variation and faced by many ethical issues. In-vitro metabolism studies are generally used to complement these data using perfused organs, tissue or cell cultures and microsomal preparations amongst which pooled human liver microsomes (HLM) have been frequently used to elucidate metabolism of SCBs [12-16]. The hepatic cell line HepG2 is often used as an initial screen as it is known to produce high reproducibility results with relatively stable enzyme concentration, although they are limited by the low-level expression of several metabolizing enzymes, including the cytochrome P450 (CYP) class of proteins $[17,18]$. The metabolic competence of HepG2 was described to be sufficient for in-vitro studies after an incubation time of at least $72 \mathrm{~h} \mathrm{[19].} \mathrm{An} \mathrm{alternative} \mathrm{to} \mathrm{this} \mathrm{was} \mathrm{the} \mathrm{use} \mathrm{of} \mathrm{a}$ microbial model with phase I and II metabolite-generating capabilities such as the zygomycete fungus, Cunninghamella elegans (C. elegans). C. elegans has the advantage of more closely mimicking mammalian metabolism [20] and has been used as a complementary model in metabolism studies of new psychoactive substances, in particular, the SCBs [21-26]. This class of fungi has an enzymatic activity to facilitate phase I and II metabolism including hydroxylation, carboxylation, dihydrodiol formation, oxidative defluorination, $\mathrm{N}$-dealkylation, glucuronidation, glucosidation, and sulfation [23, 27]. The advantages offered by the microbial transformation over other approaches include the low setting up cost, reliability and reproducibility as well as possible economical scale-up affording both phase I and II metabolites in higher yield. This scaled production is useful for further characterization of metabolites that are required to be distinguished from its isomeric species and in the identification of potentially toxic metabolites [28].

Although there were reports on the metabolism of 4F-MDMB-BINACA using in-vivo and various in-vitro models, studies were either conducted using small in-vivo sample size such as 1 to 4 samples [5, 29] or in closed environments such as forensic psychiatric wards and prisons [30]. In our study, twenty in-vivo urine samples screened positive for 4F-MDMB-BINACA intake were employed and the findings were compared to those obtained via HepG2, C. elegans, and HLM in-vitro models. The aim of this study was to assess the suitability of these in-vitro models in the in-vitro - in-vivo extrapolations and to suggest suitable urinary marker(s) for 4F-MDMB-BINACA intake.

\section{Materials and methods}

\section{Chemicals and reagents}

4F-MDMB-BINACA was provided by Health Sciences Authority (Singapore). 4F-MDMB-BINACA 3,3-dimethylbutanoic acid metabolite was purchased from Cayman Chemical (Ann Arbor, MI, USA). In-vitro metabolism study: one hundred fifty (mixed gender)- donor HLM pool, NADPH system solution A and NADPH system solution B, T75 tissue culture flasks, and 96-well cell culture plates were purchased from Corning (Corning, NY, USA); C. elegans ATCC 10028b from Cryosite Ltd. (South Granville, NSW, Australia); HepG2 cell line from CellBank (Sydney, NSW, Australia). Minimum Essential Medium-Eagle with 
Earle's BSS (MEM Eagle with EBSS) from Lonza (Basel, Switzerland); liquid chromatography - mass spectrometry (LC-MS) grade acetonitrile (ACN) from Honeywell (Muskegon, MI, USA); reagent grade dichloromethane $\left(\mathrm{CH}_{2} \mathrm{Cl}_{2}\right)$ and sodium chloride $(\mathrm{NaCl})$ from Chem-Supply (Gilman, SA, Australia); LC-MS grade formic acid from Sigma-Aldrich (St. Louis, MO, USA); glycerol, potassium dihydrogen phosphate $\left(\mathrm{KH}_{2} \mathrm{PO}_{4}\right)$ and dipotassium hydrogen phosphate $\left(\mathrm{K}_{2} \mathrm{HPO}_{4}\right)$ from Ajax Chemicals (Auburn, NSW, Australia); potato dextrose agar, glucose, peptone, and yeast extract from Oxoid Australia (Adelaide, SA, Australia). In-vivo metabolism study: chemicals for preparing reagents and buffers were obtained from Fluka, Sigma-Aldrich (St. Louis, MO, USA). Optima LC-MS grade ACN from ThermoFisher Scientific (Fair Lawn, NJ, USA); purified water was used, and all solvents used were of LC-MS grade or better; $\beta$-glucuronidase from abalone (Haliotis rufescens) from KURA Biotec (Rancho Dominguez, CA, USA); Clean Screen FASt ${ }^{\circledR}$ filter tubes (3 mL packed with $200 \mathrm{mg}$ solid-phase sorbent) from UCT, Inc (Bristol, PA, USA).

\section{Monitoring in-vitro metabolism}

\section{HepG2 liver cells}

Cryofrozen HepG2 cells were thawed and subcultured (up to passage 7) in a T75 tissue culture flask containing MEM Eagle with EBSS media supplemented with $10 \%$ heat-inactivated fetal bovine serum (HI-FBS), L-glutamine $(2 \mathrm{mM})$, penicillin:streptomycin $(100 \mathrm{U} / \mathrm{mL}: 100 \mu \mathrm{g} / \mathrm{mL})$ and non-essential amino acid solution (NEAA, 1x). The cells were cultured at $37{ }^{\circ} \mathrm{C}$ in a humidified atmosphere incubator containing $95 \%$ air and $5 \% \mathrm{CO}_{2}$, until $~ 80 \%$ confluent. The media was changed every 2 days, and when required, cells were harvested by subculture with media containing trypsin/EDTA (0.12\% trypsin/ $0.02 \%$ EDTA, w/v). The following method was adopted [31] with slight modifications: cells were seeded at a density of 9600 cells/ well onto 96-well plates overnight. Cells were treated with $120 \mu \mathrm{M}$ of 4F-MDMB-BINACA media solution $(100 \mu \mathrm{L})$ in triplicates for $72 \mathrm{~h}$. The supernatant $(60 \mu \mathrm{L})$ was transferred into a $0.5 \mathrm{~mL}$ capped tube containing ice-cold ACN $(60 \mu \mathrm{L})$. The mixture was vortex-mixed and cooled for another $15 \mathrm{~min}$ at $-30{ }^{\circ} \mathrm{C}$ before centrifuging at $10,000 \mathrm{~g}$ for $5 \mathrm{~min}$. The filtered sample $(0.22 \mu \mathrm{m}, 100 \mu \mathrm{L})$ was diluted with $\mathrm{ACN}(500 \mu \mathrm{L})$ and $10 \mu \mathrm{L}$ was subjected to liquid chromatography - quadrupole time-of-flight mass spectrometry (LC-QTOF-MS). A $2 \mu \mathrm{L}$ aliquot was also subjected to liquid chromatography-triple quadrupolemass spectrometry (LC-QQQ-MS). A control without
HepG2 and/or without 4F-MDMB-BINACA were also treated in the identical fashion.

\section{Fungus C. elegans}

C. elegans was cultured on potato dextrose agar plates at $27{ }^{\circ} \mathrm{C}$ for 5 days. The mycelia of the fungus were mixed in sterile physiological saline solution (1 plate of myce$\mathrm{lia} / 10 \mathrm{~mL}$ ). Growth medium consisting of $\mathrm{NaCl} / \mathrm{KH}_{2} \mathrm{PO}_{4} /$ yeast extract/peptone/glucose $(1: 1: 1: 1: 2, \mathrm{v} / \mathrm{v} / \mathrm{v} / \mathrm{v} / \mathrm{v})$, water and glycerol was prepared as previously described [32] and autoclaved $\left(121^{\circ} \mathrm{C}, 15 \mathrm{~min}\right)$ before use. A $1.5-\mathrm{mL}$ volume of the homogenate was inoculated into $100 \mathrm{~mL}$ of growth medium in a conical flask. The culture was incubated for $48 \mathrm{~h}$ at $26^{\circ} \mathrm{C}$ and $180 \mathrm{rpm}$ on a Multitron rotary shaker (Infors AG, Bottmingen, Switzerland). 4F-MDMB-BINACA ( $1 \mathrm{mg}$ in $0.5 \mathrm{~mL} \mathrm{ACN}$ ) was added to the conical flask in triplicate and incubated for another $72 \mathrm{~h}$. The solution was filtered in a Buchner funnel, extracted with $\mathrm{CH}_{2} \mathrm{Cl}_{2}$ $(3 \times 50 \mathrm{~mL})$. The combined extracts were evaporated to dryness using a rotary evaporator followed by a vacuum pump. The sample was reconstituted in $2 \mathrm{~mL} \mathrm{ACN}$ and filtered using a $0.22 \mu \mathrm{m}$ filter and was further diluted in ACN tenfold before injected ( $5 \mu \mathrm{L}$ ) into the LC - QTOF-MS instrument. An aliquot of the filtrate $(100 \mu \mathrm{L})$ was diluted in 900 $\mu \mathrm{L}$ water/ACN (90:10, v/v) and $2 \mu \mathrm{L}$ was also injected into the LC-QQQ-MS instrument. Control samples in the absence of $C$. elegans and/or 4F-MDMB-BINACA were also treated in the identical fashion.

\section{Pooled human liver microsomes}

The incubation mixture using 4F-MDMB-BINACA solution in $\mathrm{ACN} /$ phosphate buffer $(25 \mu \mathrm{L}$, final concentration $1.7 \mu \mathrm{M}$ in $0.03 \% \mathrm{ACN})$, phosphate buffer $(0.1 \mathrm{M}, \mathrm{pH} 7.4,855 \mu \mathrm{L})$, NADPH-A $(50 \mu \mathrm{L})$ and NADPH-B $(20 \mu \mathrm{L})$ was mixed in a $2 \mathrm{~mL}$ capped tube, to which HLM $(50 \mu \mathrm{L}=1 \mathrm{mg}$ protein $)$ was added. The mixture was vortexed mixed and incubated in triplicate at $37^{\circ} \mathrm{C}$ in a shaking water bath $(100 \mathrm{rpm})$ for $1 \mathrm{~h}$. The reaction was quenched by adding ice-cold ACN $(1 \mathrm{~mL})$ to the mixture, vortex mixed and then centrifuged at $13,000 \mathrm{~g}$ for $10 \mathrm{~min}$. The sample was filtered $(0.22 \mu \mathrm{m})$ and $5 \mu \mathrm{L}$ was injected into the LC-QTOF-MS instrument. A $100-\mu \mathrm{L}$ volume of the filtrate was diluted in 900 $\mu \mathrm{L}$ water/ACN $(90: 10, \mathrm{v} / \mathrm{v})$, and $2 \mu \mathrm{L}$ was also injected into the LC-QQQ-MS instrument. Controls samples without HLM and/or without 4F-MDMB-BINACA were also incubated and subsequently worked up under the identical conditions. 


\section{LC-QTOF-MS}

Chromatographic separation was performed on an Agilent 1290 LC system with a Poroshell 120 EC-C18 analytical column $(2.7 \mu \mathrm{m}, 75 \times 2.1 \mathrm{~mm}$; Agilent Technologies, Santa Clara, CA, USA). The mobile phase consisted of $0.1 \%$ formic acid in water (A) and $0.1 \%$ formic acid in acetonitrile (B). The gradient elution was as follows: $10 \% \mathrm{~B}$ until 2 min, increased to $40 \%$ B until 4 min, ramped to $60 \%$ B until $15 \mathrm{~min}$, and further increased to $80 \% \mathrm{~B}$ at $18 \mathrm{~min}$ and ramped down to $10 \% \mathrm{~B}$ until $19 \mathrm{~min}$ and held until $21 \mathrm{~min}$. The flow rate was $0.3 \mathrm{~mL} / \mathrm{min}$ and the column temperature was kept at $35{ }^{\circ} \mathrm{C}$. High-resolution QTOF-MS data were acquired on an Agilent 6510 Accurate Mass QTOF mass spectrometer (Agilent Technologies) equipped with dual electrospray ionization (ESI) source operated in both positive and negative ion modes, to determine accurate masses of the metabolites. The parameters were as follows: scanning mass range, $m / z, 100-700$ (MS), $m / z$ 100-600 (MS/ MS); capillary voltage, $3500 \mathrm{~V}$; nebulizer pressure, $40 \mathrm{psi}$; gas temperature, $310^{\circ} \mathrm{C}$; drying gas flow, $13 \mathrm{~L} / \mathrm{min}$; fragmentor voltage, $160 \mathrm{~V}$; collision energy $(\mathrm{CE})$ for product ion scan analysis, 10, 20 and $40 \mathrm{eV}$; skimmer voltage, $65 \mathrm{~V}$. Mass calibration was performed with the mixture provided by the manufacturer. Real-time mass calibration was enabled using the following reference masses: $\mathrm{m} / \mathrm{z} 118.086255$ and 322.048121 (positive ion mode); 112.985587 and 301.998139 (negative ion mode). Extracted ion chromatograms and product ion spectra were analysed using Agilent MassHunter Workstation Software Qualitative Analysis (version B.06.00). The criteria for metabolites elucidation were as follows: mass error of the precursor ion $\leq 5.00 \mathrm{ppm}$, mass error of product ions $\leq 20.00 \mathrm{ppm}$, consistent fragmentation pattern with the proposed structure, reasonable retention time, and absence of the specific peak in controls.

\section{LC-QQQ-MS}

Chromatographic equipment and conditions were the same as described above. MS was run in multiple reaction monitoring (MRM) mode on an Agilent 6490 Triple Quadrupole mass spectrometer with ESI source in positive ion mode (Agilent Technologies). An optimised CE $8-40 \mathrm{eV}$ was applied for each individual MRM transition. The source parameters were as follows: the capillary and nozzle voltages, 3500 and $1500 \mathrm{~V}$, respectively; the sheath gas temperature and flow set to $400{ }^{\circ} \mathrm{C}$ and $12 \mathrm{~L} / \mathrm{min}$, respectively; nebulizer pressure, $40 \mathrm{psi}$; gas temperature, $290^{\circ} \mathrm{C}$; drying gas flow, $12 \mathrm{~L} / \mathrm{min}$; fragmentor voltage, $380 \mathrm{~V}$. Mass calibration was routinely performed via check tune before analysis. Extracted ion chromatograms and MRM mass chromatograms were analysed using Agilent MassHunter Workstation Software Qualitative Analysis (version B.06.00). Percentage
(\%) peak area ratio was obtained by dividing the peak area of the detected metabolite by the peak area of the most abundant metabolite in the same sample. Major metabolites were defined as those with greater than $10 \%$ average peak area ratios.

\section{In-vivo metabolism}

\section{Authentic human urine samples}

Antemortem urine samples from routine toxicology cases $(n=20)$ confirmed with the 4F-MDMB-BINACA ester hydrolysis metabolite (4F-MDMB-BINACA 3,3-dimethylbutanoic acid metabolite) were processed and analysed for in-vivo metabolites in collaboration with the in-vitro metabolism findings. Urine sample $(500 \mu \mathrm{L})$ was incubated with $250 \mu \mathrm{L}$ of pH 5 acetate buffer $(1 \mathrm{M})$ and $50 \mu \mathrm{L}$ $\beta$-glucuronidase $(10,000$ Fisherman units $/ \mathrm{mL})$ for $1 \mathrm{~h}$ at $60{ }^{\circ} \mathrm{C}$. After incubation, the mixture was cooled at room temperature, and $150 \mu \mathrm{L}$ of purified water was added. The mixture was vortex-mixed and $500 \mu \mathrm{L}$ of this mixture and $500 \mu \mathrm{L}$ of methanol were loaded onto the Clean Screen FASt ${ }^{\circledR}$ tube. About 10-20 bar pressure was applied on the tube using a positive pressure manifold to allow all the samples to flow through the tube. The eluent was collected in a $2 \mathrm{~mL}$ sample vial for analysis.

\section{LC-Orbitrap-MS}

Chromatographic separation was achieved on a Kinetex XB-C18 column $(2.6 \mu \mathrm{m}, 100 \times 2.1 \mathrm{~mm}$; Phenomenex, Torrance, CA, USA) with an identically packed defender guard cartridge $(2.6 \mu \mathrm{m}, 10 \times 2.1 \mathrm{~mm})$. The mobile phase consisted of $10 \mathrm{mM}$ ammonium formate, $\mathrm{pH} 3(\mathrm{~A})$ and $0.1 \%$ formic acid in ACN (B). The gradient elution was as follows: $5 \% \mathrm{~B}$ until $1 \mathrm{~min}$, increased to $95 \%$ B until $10 \mathrm{~min}$, ramped to $99 \%$ $\mathrm{B}$ at $10.1 \mathrm{~min}$, held until $11.5 \mathrm{~min}$, ramped down to $5 \% \mathrm{~B}$ at $11.6 \mathrm{~min}$ and held until $13.0 \mathrm{~min}$. The flow rate was $0.5 \mathrm{~mL} /$ min for the whole run except from 10.1 to $11.5 \mathrm{~min}$ which was set at $0.8 \mathrm{~mL} / \mathrm{min}$. The identification of the metabolites was performed using liquid chromatography coupled with Thermo Scientific Q-Exactive hybrid quadrupole-Orbitrap mass spectrometer (Thermo Scientific, Fremont, CA, USA) equipped with heated electrospray ionization source (HESIII) operated in positive ionization with full MS and datadependent $\mathrm{MS}^{2}$ acquisition mode. The parameters were as follows: scanning mass range, $m / z$ 120-1000 (MS); sheath gas, 40 arbitrary units (AU); auxiliary gas, $10 \mathrm{AU}$; sweep gas, $1 \mathrm{AU}$; spray voltage, $3.5 \mathrm{kV}$; capillary temperature, $270{ }^{\circ} \mathrm{C}$; heater temperature, $425{ }^{\circ} \mathrm{C}$. The in-vitro metabolites elucidated from the cultured HepG2 cells, C. elegans and HLM were added to the compound database. Data obtained from the twenty urine samples were retrospectively analysed 
and processed using TraceFinder software based on the identification criteria of mass errors less than $\pm 5 \mathrm{ppm}$ for full MS peaks and MS/MS peaks from the theoretical mass and matching of MS/MS spectra. Percentage (\%) peak area ratios of the metabolites were similarly calculated as the in-vitro metabolism study.

\section{Results}

A total of 25 different metabolites (B1-B25) was tentatively identified and listed in Table 1 in the order of retention time (RT) with postulated biotransformation. 4F-MDMBBINACA was eluted at $12.0 \mathrm{~min}$, whilst the metabolites eluted between 5.3 and 9.6 min. Metabolites were identified according to their precursor ions, product ions, and fragmentation patterns (Fig. 1). The proposed biotransformation pathways of 4F-MDMB-BINACA are presented in Fig. 2.

\section{In-vitro metabolism}

Twenty-three, seventeen and nine metabolites were tentatively identified after incubation of 4F-MDMB-BINACA with $C$. elegans, HLM and HepG2 cells, respectively.

Ester hydrolysis of 4F-MDMB-BINACA generated 4F-MDMB-BINACA ester hydrolysis metabolite, 4F-MDMB-BINACA 3,3-dimethylbutanoic acid (B22) with further dehydrogenation (B20), monohydroxylation (B8, B9, B13) with further dehydrogenation (B5/B6, B14), oxidative defluorination (B2) with subsequent oxidation to butanoic acid (B4) and $N$-dealkylation (B1). Other metabolites retained the terminal methyl ester moiety and their biotransformation tentatively identified included: monohydroxylation (B19/B21, B23/B25) with further carbonylation (B24), dihydroxylation (B10, B12/B15), oxidative defluorination (B18) and further oxidation to butanoic acid (B17) and $N$-dealkylation (B16). Phase II glucosidation (B7) and

Table 1 In-vitro metabolites of 4F-MDMB-BINACA tentatively identified using Cunninghamella. elegans (C. elegans)(F), human liver microsomes $(\mathrm{HLM})(\mathrm{M})$ and HepG2 $(\mathrm{H})$ models

\begin{tabular}{|c|c|c|c|c|c|c|c|c|}
\hline ID & RT (min) & Biotransformation & Formula & Exact mass $[\mathrm{M}+\mathrm{H}]^{+}$ & $\mathrm{U}$ & $\mathrm{F}$ & $\mathrm{M}$ & $\mathrm{H}$ \\
\hline B1 & 5.30 & Ester hydrolysis, $N$-dealkylation & $\mathrm{C}_{14} \mathrm{H}_{17} \mathrm{~N}_{3} \mathrm{O}_{3}$ & 276.1343 & $\checkmark$ & $\checkmark$ & $\checkmark$ & $\checkmark$ \\
\hline B2 & 5.40 & Ester hydrolysis, oxidative defluorination & $\mathrm{C}_{18} \mathrm{H}_{25} \mathrm{~N}_{3} \mathrm{O}_{4}$ & 348.1918 & $\checkmark$ & $\checkmark$ & $\checkmark$ & $\checkmark$ \\
\hline B3 & 5.40 & $\begin{array}{l}\text { Ester hydrolysis, oxidative defluorination to butanoic acid, dehy- } \\
\text { drogenation }\end{array}$ & $\mathrm{C}_{18} \mathrm{H}_{21} \mathrm{~N}_{3} \mathrm{O}_{5}$ & 360.1550 & & $\checkmark$ & & \\
\hline B4 & 5.45 & Ester hydrolysis, oxidative defluorination to butanoic acid & $\mathrm{C}_{18} \mathrm{H}_{23} \mathrm{~N}_{3} \mathrm{O}_{5}$ & 362.1710 & $\checkmark$ & $\checkmark$ & $\checkmark$ & \\
\hline B5 & 5.50 & Ester hydrolysis, monohydroxylation (butyl), dehydrogenation & $\mathrm{C}_{18} \mathrm{H}_{22} \mathrm{FN}_{3} \mathrm{O}_{4}$ & 364.1667 & & $\checkmark$ & & \\
\hline B6 & 5.70 & Ester hydrolysis, monohydroxylation (butyl), dehydrogenation & $\mathrm{C}_{18} \mathrm{H}_{22} \mathrm{FN}_{3} \mathrm{O}_{4}$ & 364.1667 & & $\checkmark$ & $\checkmark$ & \\
\hline B7 & 5.80 & Monohydroxylation (indazole), glucosidation & $\mathrm{C}_{25} \mathrm{H}_{36} \mathrm{FN}_{3} \mathrm{O}_{9}$ & 542.2508 & & $\checkmark$ & & \\
\hline B8 & 5.85 & Ester hydrolysis, monohydroxylation (tert-leucine) & $\mathrm{C}_{18} \mathrm{H}_{24} \mathrm{FN}_{3} \mathrm{O}_{4}$ & 366.1824 & $\checkmark$ & $\checkmark$ & $\checkmark$ & $\checkmark$ \\
\hline B9 & 6.10 & Ester hydrolysis, monohydroxylation (butyl) & $\mathrm{C}_{18} \mathrm{H}_{24} \mathrm{FN}_{3} \mathrm{O}_{4}$ & 366.1824 & & $\checkmark$ & $\checkmark$ & \\
\hline B10 & 6.10 & Dihydroxylation (butyl) & $\mathrm{C}_{19} \mathrm{H}_{26} \mathrm{FN}_{3} \mathrm{O}_{5}$ & 396.1929 & & $\checkmark$ & & \\
\hline B11 & 6.40 & Monohydroxylation (indazole), sulfation & $\mathrm{C}_{19} \mathrm{H}_{26} \mathrm{FSN}_{3} \mathrm{O}_{7}$ & 460.1548 & & $\checkmark$ & & \\
\hline B12 & 6.60 & Dihydroxylation (indazole, butyl) & $\mathrm{C}_{19} \mathrm{H}_{26} \mathrm{FN}_{3} \mathrm{O}_{5}$ & 396.1929 & & $\checkmark$ & & \\
\hline B13 & 6.70 & Ester hydrolysis, monohydroxylation (indazole) & $\mathrm{C}_{18} \mathrm{H}_{24} \mathrm{FN}_{3} \mathrm{O}_{4}$ & 366.1824 & & $\checkmark$ & $\checkmark$ & $\checkmark$ \\
\hline B14 & 6.75 & $\begin{array}{l}\text { Ester hydrolysis, monohydroxylation (tert-leucine), dehydrogena- } \\
\text { tion }\end{array}$ & $\mathrm{C}_{18} \mathrm{H}_{22} \mathrm{FN}_{3} \mathrm{O}_{4}$ & 364.1667 & & $\checkmark$ & $\checkmark$ & \\
\hline B15 & 6.90 & Dihydroxylation (indazole, butyl) & $\mathrm{C}_{19} \mathrm{H}_{26} \mathrm{FN}_{3} \mathrm{O}_{5}$ & 396.1929 & & $\checkmark$ & & \\
\hline B16 & 7.10 & $N$-Dealkylation & $\mathrm{C}_{15} \mathrm{H}_{19} \mathrm{~N}_{3} \mathrm{O}_{3}$ & 290.1499 & & $\checkmark$ & $\checkmark$ & $\checkmark$ \\
\hline B17 & 7.30 & Oxidative defluorination to butanoic acid & $\mathrm{C}_{19} \mathrm{H}_{25} \mathrm{~N}_{3} \mathrm{O}_{5}$ & 376.1867 & $\checkmark$ & $\checkmark$ & $\checkmark$ & $\checkmark$ \\
\hline B18 & 7.35 & Oxidative defluorination & $\mathrm{C}_{19} \mathrm{H}_{27} \mathrm{~N}_{3} \mathrm{O}_{4}$ & 362.2074 & $\checkmark$ & $\checkmark$ & $\checkmark$ & $\checkmark$ \\
\hline B19 & 7.70 & Monohydroxylation (butyl) & $\mathrm{C}_{19} \mathrm{H}_{26} \mathrm{FN}_{3} \mathrm{O}_{4}$ & 380.1980 & & $\checkmark$ & $\checkmark$ & \\
\hline B20 & 7.90 & Ester hydrolysis, dehydrogenation & $\mathrm{C}_{18} \mathrm{H}_{22} \mathrm{FN}_{3} \mathrm{O}_{3}$ & 348.1718 & $\checkmark$ & $\checkmark$ & $\checkmark$ & $\checkmark$ \\
\hline B21 & 8.10 & Monohydroxylation (butyl) & $\mathrm{C}_{19} \mathrm{H}_{26} \mathrm{FN}_{3} \mathrm{O}_{4}$ & 380.1980 & & $\checkmark$ & $\checkmark$ & \\
\hline B22 & 8.30 & Ester hydrolysis & $\mathrm{C}_{18} \mathrm{H}_{24} \mathrm{FN}_{3} \mathrm{O}_{3}$ & 350.1874 & $\checkmark$ & $\checkmark$ & $\checkmark$ & $\checkmark$ \\
\hline B23 & 8.50 & Monohydroxylation (indazole) & $\mathrm{C}_{19} \mathrm{H}_{26} \mathrm{FN}_{3} \mathrm{O}_{4}$ & 380.1980 & & & $\checkmark$ & \\
\hline B24 & 9.00 & Carbonylation & $\mathrm{C}_{19} \mathrm{H}_{24} \mathrm{FN}_{3} \mathrm{O}_{4}$ & 378.1824 & & $\checkmark$ & & \\
\hline B25 & 9.60 & Monohydroxylation (indazole) & $\mathrm{C}_{19} \mathrm{H}_{26} \mathrm{FN}_{3} \mathrm{O}_{4}$ & 380.1980 & & & $\checkmark$ & \\
\hline B0 & 12.00 & 4F-MDMB-BINACA & $\mathrm{C}_{19} \mathrm{H}_{26} \mathrm{FN}_{3} \mathrm{O}_{3}$ & 364.2031 & & $\checkmark$ & $\checkmark$ & $\checkmark$ \\
\hline
\end{tabular}

Mass error of the precursor ion $\leq 5.00 \mathrm{ppm}$ and mass error of product ions $\leq 20.00 \mathrm{ppm}$

$I D$ identity, $R T$ retention time, $U$ in-vivo urine sample 

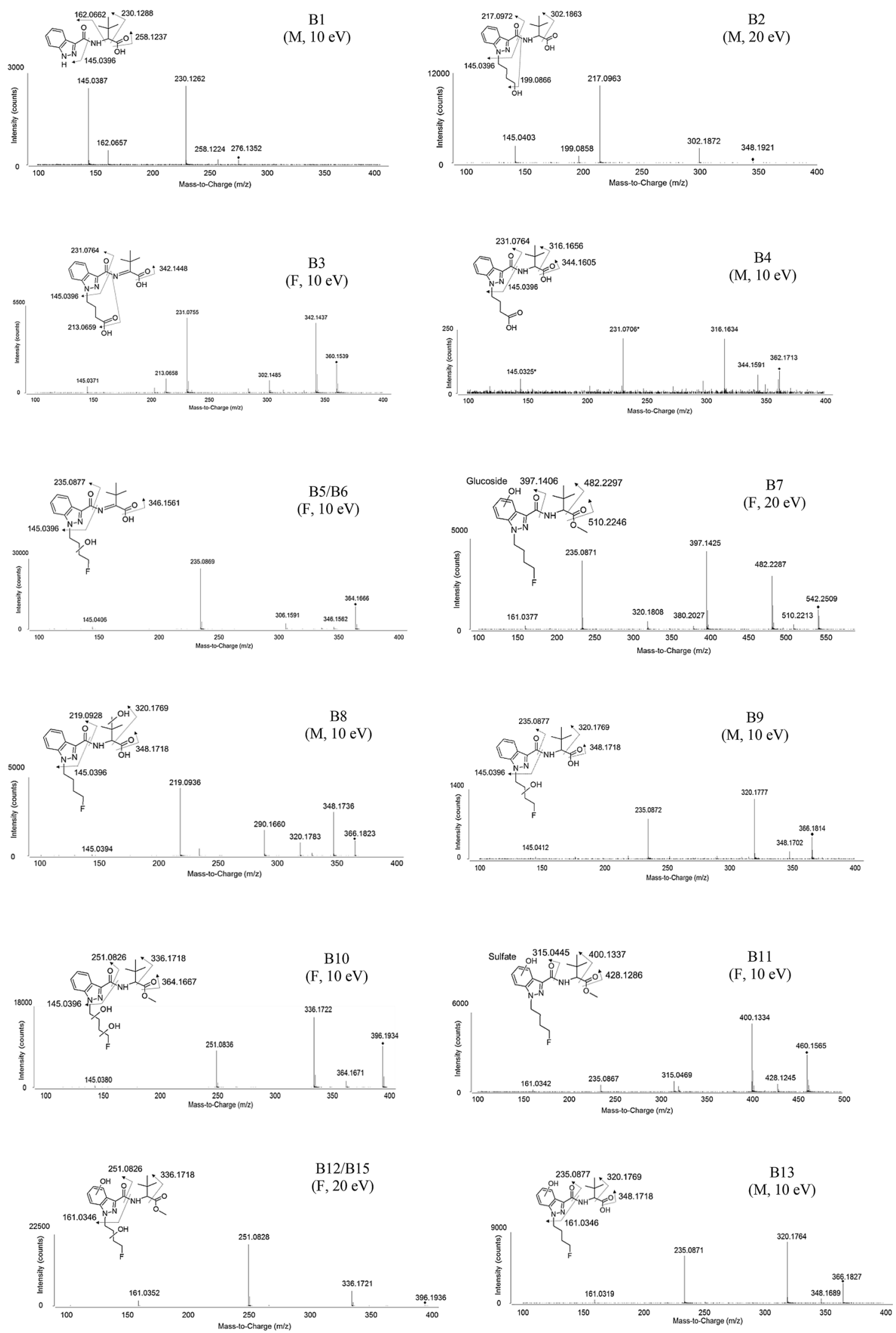

Fig. 1 Product ion spectra and tentative structures with the suggested fragmentation patterns from the three in-vitro models: Cunninghamella elegans (C. elegans)(F), human liver microsomes (HLM)(M)

and HepG2 $(\mathrm{H})$. The brackets indicate product ion spectra obtained from one of the in-vitro model with collision energy 10 or $20 \mathrm{eV}$. The exact position of the hydroxylation was not determined 

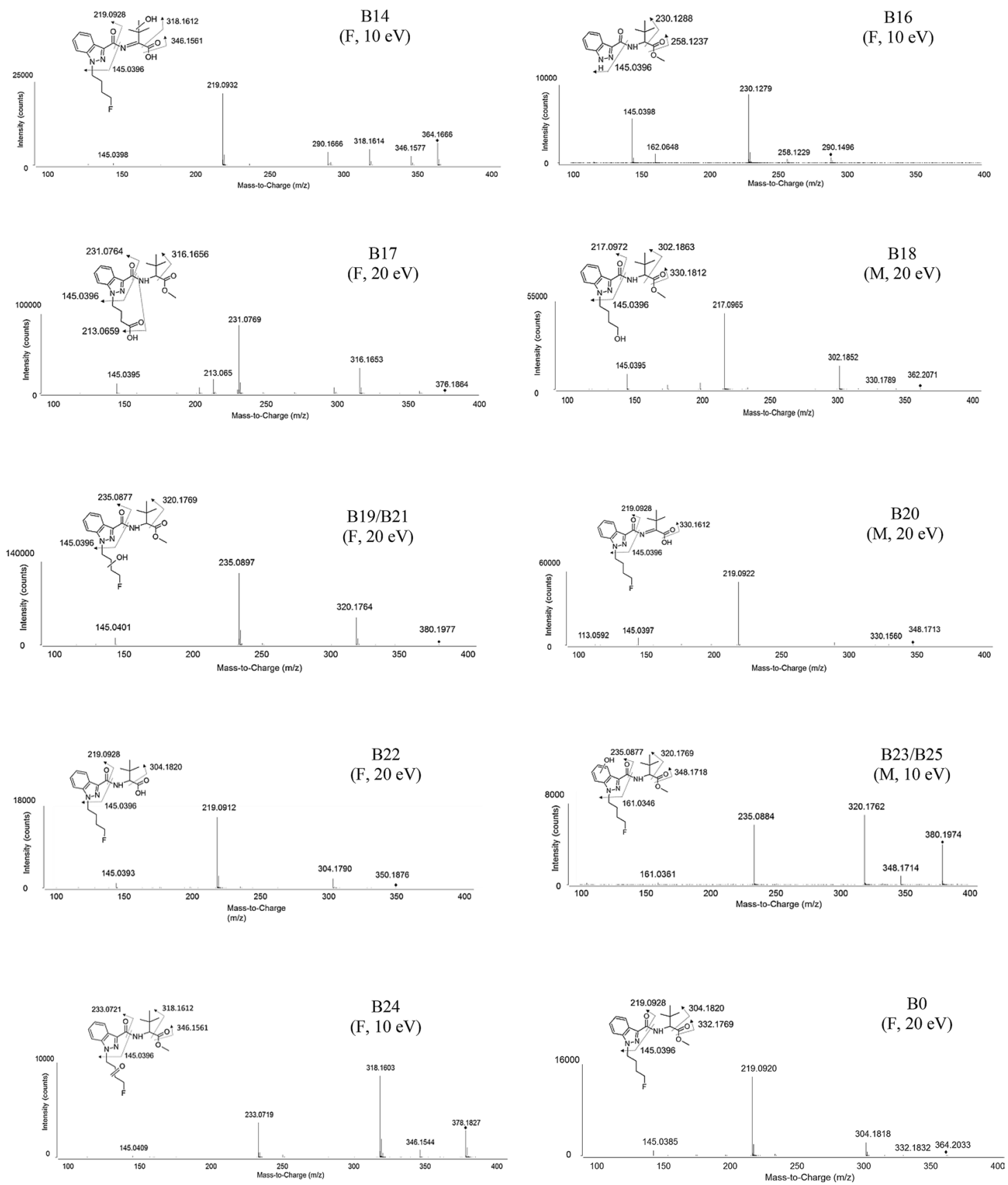

Fig. 1 (continued)

sulfation (B11) metabolites were only detected in the $C$. elegans model. The tentative structure elucidation of these various metabolites from the different models examined was constructed and shown below.

\section{Phase I biotransformation}

Ester hydrolysis, dehydrogenation, hydroxylation, oxidation and dealkylation

All the below explanations for transformations into metabolites are based on the data shown in Fig. 1 and on probable metabolic pathways shown in Fig. 2. 4F-MDMB-BINACA was hydrolysed via ester hydrolysis forming the 4F-MDMBBINACA ester hydrolysis metabolite (B22). The product ion 


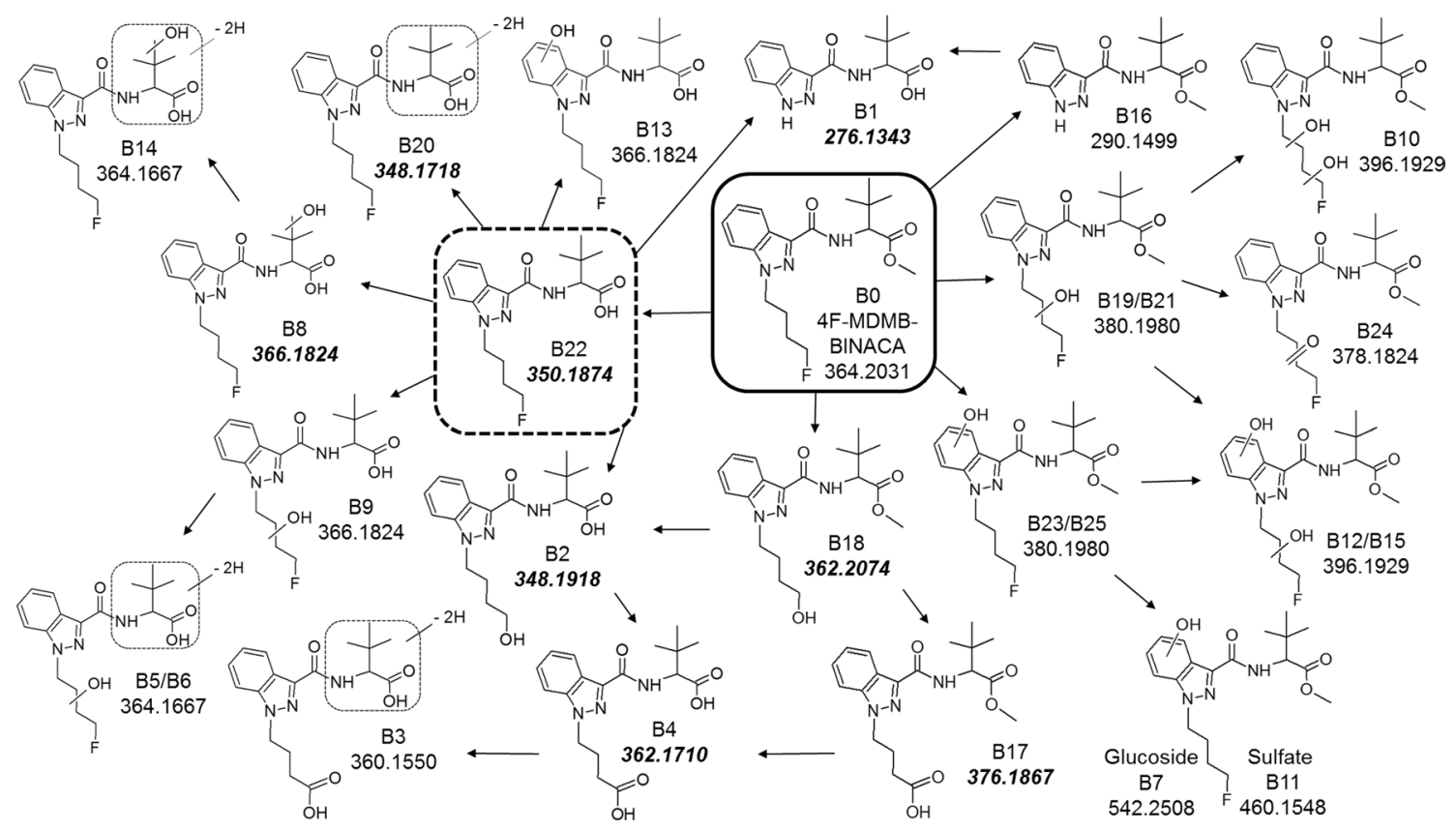

Fig. 2 Proposed metabolic pathways of 4F-MDMB-BINACA. In-vivo metabolites were in italics and bold. The exact position of the hydroxyl moiety of the metabolites was not determined

spectrum was very similar to 4F-MDMB-BINACA $(\mathrm{m} / \mathrm{z}, 304$, $219,145)$. The precursor ion, $m / z 350$ showed a loss of $14 \mathrm{Da}$ explaining the hydrolysis of methyl ester from 4F-MDMBBINACA. B20 showed a similar product ion fragmentation pattern to that of $\mathrm{B} 22(\mathrm{~m} / \mathrm{z}, 219,145)$ and additional loss of $2 \mathrm{Da}$ from $\mathrm{m} / \mathrm{z} 350$, indicating ester hydrolysis and further dehydrogenation at the tert-leucine moiety.

Metabolites identified at $m / z 366$ (B8, B9, B13), which was $16 \mathrm{Da}$ higher than the 4F-MDMB-BINACA ester hydrolysis metabolite (B22), confirmed monohydroxylation upon ester hydrolysis. The presence of the product ion $\mathrm{m} / \mathrm{z}$ 320 , likely formed from a loss of carbon dioxide, indicated monohydroxylation at the tert-leucine in B8 $(\mathrm{m} / \mathrm{z} 219)$, butyl side chain in B9 $(\mathrm{m} / \mathrm{z} 145)$ and indazole moiety in B13 $(\mathrm{m} / \mathrm{z}$ $161)$. The precursor ion, $m / z 364$ (B14, B5/B6) had a loss of 2 Da from $m / z, 366$ indicated further dehydrogenation of the ester hydrolysis plus monohydroxylated metabolites. Dehydrogenation occurred at the tert-leucine moieties for both the monohydroxylated metabolites (B5/B6, B14), indicated by the presence of product ions $m / z 235 / 346$ and $m / z 219 / 346$, respectively. Similarly, precursor ion identified at $\mathrm{m} / \mathrm{z} 380$ (B19/B21, B23/B25) was 16 Da higher than the 4F-MDMBBINACA, indicating monohydroxylation at the butyl side chain (B19/B21) and indazole (B23/B25) moieties with product ions $m / z 145$ and 161, respectively. The metabolite (B24) identified at $m / z, 378$ had very similar product ion spectra with B19/B21 except it was 2 Da lower (equating to the loss of 2 hydrogen atoms) while retaining the same product ion $m / z, 145$, indicating carbonylation at the butyl side chain moiety.

The precursor ion $m / z 396$ (B10, B12/B15) was $32 \mathrm{Da}$ higher than the parent drug, 4F-MDMB-BINACA, suggesting the addition of two hydroxy groups. The product ion $\mathrm{m} / \mathrm{z} 336$ (loss of methyl ester moiety) further confirmed the presence of dihydroxylated metabolites. The presence of product ion $\mathrm{m} / \mathrm{z} 251$ at B10 and B12/B15 was explained by noting that hydroxylation occurred at the indazole/butyl side chain moieties and not at the tert-leucine moiety. B10 showed product ion $\mathrm{m} / \mathrm{z} 145$ indicating intact indazole moiety and suggested that dihydroxylation occurred at the butyl side chain moiety. The presence of product ion $\mathrm{m} / \mathrm{z}$ 161 in B12/B15, indicated that hydroxylation occurred at the indazole moiety, whilst the second hydroxylation site must be assigned at the butyl side chain moiety.

The precursor ion $m / z, 348$ and product ion detected at $m / z 217$ (B2) identified was 2 Da less than the 4F-MDMBBINACA ester hydrolysis metabolite (B22), indicating oxidative defluorination (loss of fluorine with addition of hydroxy group). Product ions detected at $\mathrm{m} / \mathrm{z} 302,217$, and 145 (B2) confirmed that tert-leucine and indazole moieties remained unchanged, leading to the structure elucidation of a hydroxy-functional group at the 4-position of the butyl side chain by oxidative defluorination. Oxidation of 4'-hydroxybutyl moiety in B2 led to formation of 4'-carboxybutyl metabolite (B4) having a precursor ion of $m / z$ 362 , which was $14 \mathrm{Da}$ higher than the $\mathrm{m} / \mathrm{z}$ for B2 (loss of two hydrogen atoms with addition of a carbonyl group). 
The precursor ion $\mathrm{m} / \mathrm{z} 360$ and product ion detected at $\mathrm{m} / \mathrm{z} 342$ (B3) identified was 2 Da less than precursor ion at $m / z 362$ (B4) indicated further dehydrogenation at the tert-leucine moiety.

The precursor ion $m / z 276$ (B1) detected, which was 74 Da lower than that for the 4F-MDMB-BINACA ester hydrolysis metabolite (B22), indicated $N$-dealkylation of B22. The product ion of B1 detected at $\mathrm{m} / \mathrm{z} 230$ was assigned as a neutral loss of $\mathrm{HCOOH}$.

\section{$N$-Dealkylation}

The precursor ion $m / z 290$ (B16) which was 74 Da lower than the corresponding $\mathrm{m} / \mathrm{z}$ for $4 \mathrm{~F}-\mathrm{MDMB}-\mathrm{BINACA}$, indicated $N$-dealkylation of 4F-MDMB-BINACA. Product ions $\mathrm{m} / z, 258,230$ and 145 showed loss of a single methoxy group, methyl ester and indazole carbonyl fragment, respectively. Taken together these data further confirmed the structure elucidation of B16.

\section{Oxidative defluorination and oxidation}

Metabolite B18 with precursor ion at $m / z, 362$ and product ions at $\mathrm{m} / \mathrm{z} 330,302,217$ were consecutively 2 Da less than that of the precursor and product ions of 4F-MDMBBINACA, being indicative of oxidative defluorination. Further oxidation of 4'-hydroxybutyl moiety of B 18 resulted in 4'-carboxybutyl, B17 ( $\mathrm{m} / \mathrm{z} 376)$ having product ions $m / z, 316,231$ and 213. The product ions at $\mathrm{m} / \mathrm{z} 316$ and 231 in B17 were 14 Da higher than that of product ions 302 and 217, respectively, in B18, consistent with the formation of a butanoic acid metabolite.

\section{Phase II biotransformation}

\section{Glucosidation}

Precursor ion, $m / z 542$ (B7) resulted from glucosidation of B23/B25. Both have product ions $\mathrm{m} / \mathrm{z} 320,235$ and 161 . The product ion detected at $m / z, 380$ was produced by neutral loss of $162 \mathrm{Da}$ (glucoside).

\section{Sulfation}

Precursor ion, $m / z 460$ (B11) detected was a result of sulfation of intermediate metabolite similar to B23/B25. The product ion detected at $\mathrm{m} / \mathrm{z} 235$, indicating loss of sulfate, confirmed the identity of the sulfation metabolite.

\section{Comparison among the in-vitro models}

The major in-vitro phase I metabolites ( $>10 \%$ peak area ratio) tentatively identified were monohydroxylation at the butyl side chain and oxidative defluorination to butanoic acid for the C. elegans model. HLM model produced four major metabolites: ester hydrolysis with/without dehydrogenation, monohydroxylation at indazole moiety and oxidative defluorination. HepG2 model only produced one major metabolite (B22) which was the ester hydrolysis of 4F-MDMB-BINACA (Table 2). Although the metabolite ranking may differ in all the three models, four common metabolites amongst the seven most abundant metabolites were identified (Fig. 3 a-d): ester hydrolysis (B22, $\mathrm{m} / \mathrm{z} 350$ ), ester hydrolysis followed by dehydrogenation (B20, $\mathrm{m} / \mathrm{z}$ 348), oxidative defluorination (B18, $\mathrm{m} / \mathrm{z} 362)$ and further oxidation to butanoic acid (B17, $\mathrm{m} / \mathrm{z} 376)$.

Table 2 Comparison of the in-vitro metabolites from the three models $(n=3)$ with the in-vivo metabolites obtained from authentic human urine samples $(n=20)$

\begin{tabular}{|c|c|c|c|c|c|}
\hline \multirow[t]{2}{*}{ ID } & \multirow[t]{2}{*}{ Biotransformation } & \multirow[t]{2}{*}{$\begin{array}{l}\text { In-vivo metabolites } \% \text { peak } \\
\text { area ratio (sample size) }\end{array}$} & \multicolumn{3}{|c|}{$\begin{array}{l}\text { In-vitro metabolites \% peak area } \\
\text { ratio }\end{array}$} \\
\hline & & & C. elegans & HLM & HepG2 \\
\hline B22 & Ester hydrolysis & $100(20 / 20)$ & 2.3 & 100 & 100 \\
\hline B20 & Ester hydrolysis, dehydrogenation & $1.7-9.4(18 / 20)$ & 3.8 & 70 & 1.4 \\
\hline B4 & Ester hydrolysis, oxidative defluorination to butanoic acid & $1.5-27(16 / 20)$ & 0.016 & 0.25 & ND \\
\hline B8 & Ester hydrolysis, monohydroxylation (tert-leucine) & $3.7-14(16 / 20)$ & 0.067 & 1.9 & 0.14 \\
\hline B1 & Ester hydrolysis, $N$-dealkylation & $0.65-29(15 / 20)$ & 0.003 & 0.73 & 6.4 \\
\hline B18 & Oxidative defluorination & $1.4-5.4(13 / 20)$ & 2.1 & 10 & 1.0 \\
\hline B2 & Ester hydrolysis, oxidative defluorination & $2.0-11(12 / 20)$ & 0.005 & 0.67 & 1.5 \\
\hline B17 & Oxidative defluorination to butanoic acid & $0.26-1.4(7 / 20)$ & 76 & 8.9 & 0.57 \\
\hline
\end{tabular}

Major in-vitro metabolites (not less than $10 \%$ peak area ratio) are in italic and bold

$N D$ not detected 
Fig. 3 a In-vitro metabolites observed in common among respective seven most abundant metabolites in b C. elegans, c HLM and d HepG2 models. C. elegans, HLM and HepG2 are represented by horizontal, diagonal and vertical bar lines, respectively. Error bars reflect the relative abundance variation of a specific metabolite within the analysed group $(n=3)$ a

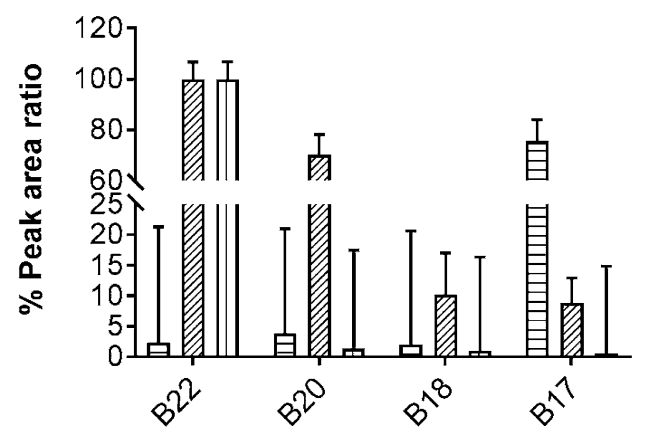

C

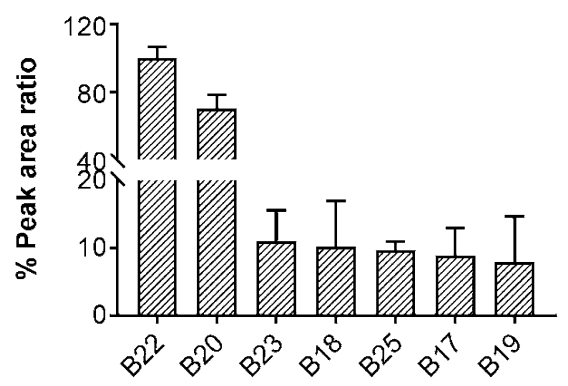

b

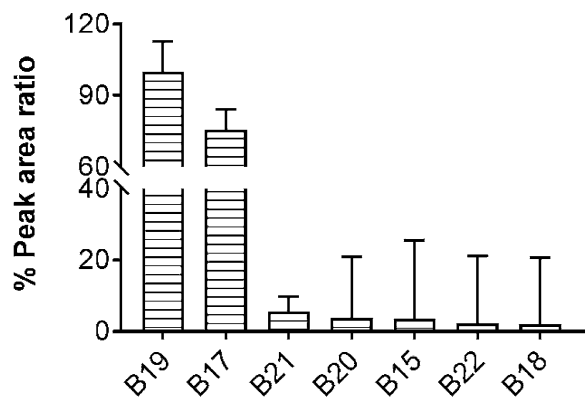

d

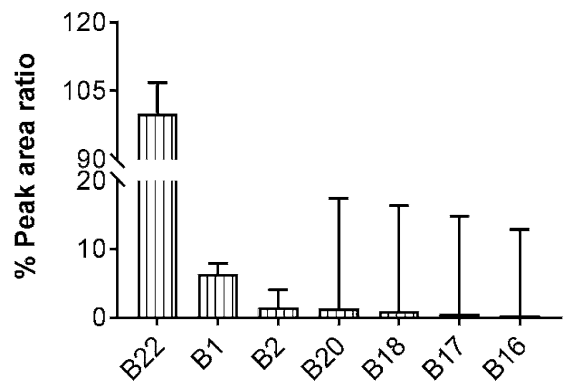

\begin{tabular}{|l|l|}
\hline ID & Biotransformation \\
\hline B1 & Ester hydrolysis, $N$-dealkylation \\
\hline B2 & Ester hydrolysis, oxidative defluorination \\
\hline B15 & Dihydroxylation (indazole, butyl) \\
\hline B16 & $N$-dealkylation \\
\hline B17 & Oxidative defluorination to butanoic acid \\
\hline B18 & Oxidative defluorination \\
\hline B19 & Monohydroxylation (butyl) \\
\hline B20 & Ester hydrolysis, dehydrogenation \\
\hline B21 & Monohydroxylation (butyl) \\
\hline B22 & Ester hydrolysis \\
\hline B23 & Monohydroxylation (indazole) \\
\hline B25 & Monohydroxylation (indazole) \\
\hline & \\
\hline
\end{tabular}

\section{In-vivo metabolism}

A total of eight metabolites were detected in the analysed human urine samples (Tables 2, 3). The average \% peak area ratio of the urine metabolites from twenty urine samples were tabulated and compared to that of the in-vitro metabolites (Table 2).

Ester hydrolysis biotransformation constituted the dominant metabolic pathway for the in-vivo metabolism in human urine samples. The prevalence of the tentatively identified metabolites was as follows: 4F-MDMB-BINACA ester hydrolysis metabolite (B22, $\mathrm{m} / \mathrm{z}$ 350, 20/20 samples) followed by further dehydrogenation (B20, $m / z$ 348, 18/20 samples). Both ester hydrolysis followed by oxidative defluorination to butanoic acid (B4, $m / z, 362)$ and monohydroxylation at tert-leucine moiety $(\mathrm{B} 8, \mathrm{~m} / \mathrm{z}, 366)$ metabolites were found in 16/20 urine samples (Table 2). Ester hydrolysis followed with $\mathrm{N}$-dealkylation (B1, $\mathrm{m} / \mathrm{z}, 276)$ metabolite was found in $15 / 20$ urine samples. Oxidative defluorination $(\mathrm{B} 18, \mathrm{~m} / \mathrm{z}$ 362 ) and ester hydrolysis of oxidative defluorination (B2, $m / z$ 348) metabolites were detected in 13/20 and 12/20 urine samples, respectively. Oxidative defluorination to butanoic acid metabolite (B17, $\mathrm{m} / \mathrm{z}$ 376) was detected in the least number of urine samples (7/20 samples). Only 3/20 urine samples were identified with the parent drug, 4F-MDMBBINACA, highlighting the risk of analysing only parent ions and emphasizing the importance of metabolite identification in the forensic and clinical setting. The metabolite ranking was different for different individual urine samples.

The analysed urine samples (17/20 samples) were also detected with other SCBs such as methyl 2-([1-(5-fluoropentyl)-1H-indole-3-carbonyl] 
Table 3 In-vivo urinary profile of individual urine samples that contain only 4F-MDMBBINACA metabolites

\begin{tabular}{lllll}
\hline ID & Biotransformation & \multicolumn{2}{l}{$\%$ Peak area ratio (ranking) } & \\
\cline { 4 - 5 } & & Sample 1 & Sample 2 & Sample 3 \\
\hline B22 & Ester hydrolysis & $100(1)$ & $100(1)$ & $100(1)$ \\
B20 & Ester hydrolysis, dehydrogenation & $4.9(4)$ & ND & $4.2(4)$ \\
B4 & $\begin{array}{l}\text { Ester hydrolysis, oxidative defluorination to } \\
\text { butanoic acid }\end{array}$ & $12(2)$ & ND & $6.8(2)$ \\
B8 & $\begin{array}{l}\text { Ester hydrolysis, monohydroxylation } \\
\text { tert-leucine) }\end{array}$ & $7.4(3)$ & ND & $5.2(3)$ \\
B1 & $\begin{array}{l}\text { Ester hydrolysis, } \\
N \text {-dealkylation }\end{array}$ & ND & ND & $0.81(7)$ \\
B18 & $\begin{array}{l}\text { Oxidative } \\
\text { defluorination }\end{array}$ & $2.2(5)$ & ND & $2.3(6)$ \\
B2 & $\begin{array}{l}\text { Ester hydrolysis, } \\
\text { oxidative defluorination }\end{array}$ & ND & ND & $2.7(5)$ \\
B17 & $\begin{array}{l}\text { Oxidative defluorination } \\
\text { to butanoic acid }\end{array}$ & $0.55(6)$ & ND & ND \\
B0 & 4F-MDMB-BINACA & 0.020 & ND & ND \\
\hline
\end{tabular}

amino)-3,3-dimethylbutanoate (5F-MDMB-PICA), methyl 3,3-dimethyl-2-[1-(pent-4-en-1-yl)-1H-indazole-3-carboxamido] butanoate (MDMB-4en-PINACA), methyl [1-(4-fluorobenzyl)-1 $H$-indazole-3-carbonyl]valinate (MMB-FUBINACA), 5F-MDMB-PINACA and other drugs such as methamphetamine, $N$-methyl-1-(3,4-methylenedioxyphenyl)propan-2-amine (MDMA) and ketamine (data not shown). Three out of twenty urine samples were found to contain only metabolites of 4F-MDMB-BINACA (Table 3). From these three samples, sample 2 contained only an ester hydrolysis metabolite $(\mathrm{m} / \mathrm{z}$ 350). Both samples 1 and 3 have similar first four metabolites in terms of $\%$ peak area ratio ranking: (1) ester hydrolysis (ion detected at $m / z$ 350, B22), (2) ester hydrolysis, oxidative defluorination to butanoic acid (ion detected at $m / z$ 362, B4), (3) ester hydrolysis, monohydroxylation (ion detected at $m / z, 366$, B8) and (4) ester hydrolysis plus dehydrogenation (ion detected at $\mathrm{m} / \mathrm{z} 348$, B20).

\section{Discussion}

Glucosides (B7) and sulfate (B11) phase II metabolites of 4F-MDMB-BINACA were tentatively identified in the $C$. elegans model. Glucosides and sulfate metabolites have been reported with other SCBs where $C$. elegans was employed as the in-vitro model [24]. Sulfate and glucuronide phase II metabolites of 4F-MDMB-BINACA was identified by Wagmann et al. [29]. However, glucuronides of 4F-MDMBBINACA were not observed in the $C$. elegans model, which is consistent with findings from previous studies [23, 24]. Rydevik et al. [33] described an easy method to generate endogenous glucuronide conjugates from glucosides using free radical tetramethylpiperidinyl-1-oxy, hence proving
C. elegans' relevance in mammalian phase II metabolism. Nevertheless, phase I metabolites are usually better marker metabolites compared to phase II metabolites because of its higher mass spectrometry responses and stability over time [34].

The most prevalent and abundant in-vivo metabolite was that derived from the hydrolysis of metabolically labile terminal methyl ester moiety via a two-step reaction mechanism by the carboxylesterases (CES) [35]. The finding is similar to that obtained by Krotulski et al. [5], Haschimi et al. [30] and Wagmann et al. [29]. This outcome was anticipated since CES-mediated hydrolysis is commonly reported as the major metabolic pathway among the SCBs impacting the terminal ester group [36]. Similar to the in-vivo findings, 4F-MDMB-BINACA ester hydrolysis (B22) was the major metabolite for both HepG2 and HLM models, consistent with the known hydrolytic activity of CES reported [37]. Wagmann et al. [29] established that ester hydrolysis of 4F-MDMB-BINACA is catalysed by the CES1 isoform, in agreement with the preference of CES1 in metabolizing ester substrates that contain a small alcohol group and a bulky acyl group [35]. As CES constitutes the major metabolic pathways for the metabolism of 4F-MDMB-BINACA, interindividual CES1 variability due to genetic polymorphism cannot be overlooked [38].

Ester hydrolysis plus dehydrogenation metabolite (B20) was the second most abundant major metabolite in the HLM model, similar to the in-vivo findings where it is the second most prevalent metabolite detected in 18/20 urine samples (Table 2). Ester hydrolysis with dehydrogenation formed invivo in this study was also reported among other indazole carboxamide type SCBs with tert-leucine methyl ester moieties such as 5F-MDMB-PINACA [15] and MDMB-4enPINACA [39, 40]. This biotransformation was reported by 
both Krotulski et al. [5]. and Haschimi et al. [30]. However, it was proposed by Wagmann et al. [29] that this metabolite was more likely a lactone product formed after hydroxylation of the tert-leucine moiety, with or without former ester hydrolysis of 4F-MDMB-BINACA, based upon the presence of fragments with $m / z 320\left(\mathrm{C}_{17} \mathrm{H}_{23} \mathrm{FN}_{3} \mathrm{O}_{2}{ }^{+}\right)$and $\mathrm{m} / 2113\left(\mathrm{C}_{6} \mathrm{H}_{9} \mathrm{O}_{2}^{+}\right)$, a 4,4-dimethyl-2-oxotetrahydrofuran3 -ylium ion in positive ionisation mode, which appears to be more easily formed from lactone, and the absence of these lactone metabolites in negative ionisation mode due to its non-ionizable nature in contrast to ionizable carboxylic acids. Although this proposal sounds plausible, it is not without question. The presence of $m / z, 330\left(\mathrm{C}_{18} \mathrm{H}_{21} \mathrm{FN}_{3} \mathrm{O}_{2}{ }^{+}\right)$ in their spectrum of "lactone" indicates loss of $\mathrm{H}_{2} \mathrm{O}$ from the precursor ion and similarly the presence of $\mathrm{m} / \mathrm{z} 332$ $\left(\mathrm{C}_{18} \mathrm{H}_{23} \mathrm{FN}_{3} \mathrm{O}_{2}^{+}\right)$due to loss of $\mathrm{H}_{2} \mathrm{O}$ was observed for ester hydrolysis metabolite, and yet such a $\mathrm{H}_{2} \mathrm{O}$ loss fragment ion was not observed for the parent molecule retaining a methyl ester moiety. These results suggest that this compound is more likely to have a carboxylic acid than an ester, which contradicts the aforementioned reasoning by Wagmann et al. [29]. The same authors also proposed lactone formation for the B3 and B14 metabolites. Careful examination of results in this study showed the presence of the ion at $\mathrm{m} / z .113$ in very low signal intensity for B20 but not found for both B3 and B14 product ion spectra. Moreover, B3 and B14 were detected in the negative ESI mode but not for B20 (Table 4). Based upon these findings, it appeared that both lactone and dehydrogenation metabolites were present, but since mass spectral results can be inconclusive and indeed seemed to be contradictory in this case, further comprehensive structural analysis by nuclear magnetic resonance (NMR) spectroscopy is needed for confirmation.

Oxidative defluorination metabolite (B18), the second major metabolite (\% peak area ratio > 10) in HLM model was detected as the third least prevalent metabolite in the in-vivo samples. Oxidative defluorination with subsequent butanoic acid formation (B17) metabolite, the second major metabolite after monohydroxylation in the $C$. elegans model was the least prevalent metabolite found among the 20 urine samples. Fluoro-substituted SCBs at the 5-pentyl position of pentylindole/pentylindazole moiety was found to give rise to predominantly 5-hydroxypentyl and 5-pentanoic acid metabolites [41]. However, oxidative defluorination with (B17) or without further butanoic acid (B18) metabolite was found only in 7 and 13 urine samples, respectively. The B18 metabolite was ranked third by Haschimi et al. [30], whilst B17 was ranked second by Krotulski et al. [5] in terms of relative peak area abundance. One possible explanation for the differences was that both Haschimi et al. [30] and Krotulski et al. [5] used relative peak area abundance for the ranking, but for this study, the metabolite prevalence in the urine samples was employed instead. The major invitro metabolites discussed above: B22, B20, B18 and B17 (Table 2) were also the four common metabolites identified among the seven most abundant metabolites for each model (Fig. 3 a-d), demonstrating complementary in-vitro findings that may assist in further streamlining the selection of suitable urinary marker (s).

As most of the urine samples obtained in this study were found to contain other SCBs' metabolites and other drugs, the urinary profile of the three individual urine samples that encompass 4F-MDMB-BINACA metabolites exclusively (Table 3 ) is worth considering when selecting a suitable urinary marker. The four most abundant metabolites identified (B22, B4, B8 and B20) among the three urine samples almost coincided with the top four most prevalent metabolites found in the 20 urine samples. This indicated that the phase I metabolism of 4F-MDMB-BINACA are unlikely to be affected significantly by polydrug intake. Wagmann et al. [29] identified CYP3A5, CYP3A4, CYP1A2, CYP2C8 and CYP2C19 involvement in the phase I metabolism of

Table 4 Product ion spectra of B3 and B14 metabolites obtained in the negative electrospray ionization mode

\begin{tabular}{|c|c|c|c|c|c|c|c|}
\hline ID & $\mathrm{RT}(\min )$ & Biotransformation & $\begin{array}{l}\text { Precursor ion } \\
\text { exact mass } \\
{[\mathrm{M}-\mathrm{H}]^{-}}\end{array}$ & $\mathrm{CE}(\mathrm{eV})$ & Formula & $\begin{array}{l}\text { Product ions } \\
\text { exact mass }\left[\mathrm{M}^{-}\right]\end{array}$ & $\begin{array}{l}\text { Product } \\
\text { ions } \\
\text { mass } \\
\text { error } \\
\text { (ppm) }\end{array}$ \\
\hline B3 & 5.42 & $\begin{array}{l}\text { Ester hydrolysis, oxidative } \\
\text { defluorination to butanoic } \\
\text { acid, dehydrogenation }\end{array}$ & 358.1408 & 10 & $\begin{array}{l}\mathrm{C}_{14} \mathrm{H}_{14} \mathrm{~N}_{3} \mathrm{O}_{3} \\
\mathrm{C}_{7} \mathrm{H}_{8} \mathrm{NO}_{3} \\
\mathrm{C}_{7} \mathrm{H}_{5} \mathrm{~N}_{2}\end{array}$ & $\begin{array}{l}272.1041 \\
154.0510 \\
117.0458\end{array}$ & $\begin{array}{l}3.3 \\
2.6 \\
4.3\end{array}$ \\
\hline B14 & 6.75 & $\begin{array}{l}\text { Ester hydrolysis, monohy- } \\
\text { droxylation (tert-leucine), } \\
\text { dehydrogenation }\end{array}$ & 362.1522 & 20 & $\begin{array}{l}\mathrm{C}_{17} \mathrm{H}_{21} \mathrm{FN}_{3} \mathrm{O}_{2} \\
\mathrm{C}_{12} \mathrm{H}_{13} \mathrm{FN}_{3} \mathrm{O} \\
\mathrm{C}_{11} \mathrm{H}_{12} \mathrm{FN}_{2} \\
\mathrm{C}_{11} \mathrm{H}_{11} \mathrm{~N}\end{array}$ & $\begin{array}{l}318.1623 \\
234.1048 \\
191.0990 \\
171.0928\end{array}$ & $\begin{array}{l}12 \\
4.3 \\
-11 \\
-2.3\end{array}$ \\
\hline
\end{tabular}

$C E$ collision energy

Mass error of the precursor ion $\leq 5.00 \mathrm{ppm}$ 
4F-MDMB-BINACA and also excluded the possibility of a single CYP enzyme that may impact the in-vivo biotransformation of 4F-MDMB-BINACA.

It was difficult to assign a ranking to the in-vivo metabolites based upon \% peak area ratio because wide inter-individual variability was observed among the metabolites. The $\%$ peak area abundance ratio of metabolites detected in the urine samples are often affected by numerous factors such as drug intake behaviour (intake route, amount of drug and intake frequency), time from last drug intake [16] and metabolic stability. It should be noted that \% peak area abundance ratios do not necessarily reflect absolute concentrations due to differences in ionization capacity and matrix effects bias for each metabolite. The exact ranking of metabolites with respect to their peak area abundance would require further validated quantification of the metabolites. Moreover, genetic makeup, physiological conditions (age, gender and ethnicity), environmental influences (diet) and pathological factors (liver diseases, diabetes, and obesity) would further complicate the metabolism of drugs. Thus, identification of the relevant urinary markers was based primarily upon the prevalence of the in-vivo metabolites instead of the metabolites ranking that was based upon \% peak area abundance ratio. The urinary marker selected would also be preferably found in all three in-vitro models especially in the C. elegans model where metabolite production could be scaled up to allow isolation and characterization of the metabolites.

Based upon the two criteria and the above findings suggested relevant in-vivo urinary markers were ester hydrolysis, $m / z, 350$ (B22, in 20/20 samples) and ester hydrolysis followed by dehydrogenation, $\mathrm{m} / z 348$ (B20, in 18/20 samples) metabolites. Ester hydrolysis with subsequent oxidative defluorination to butanoic acid metabolite $(\mathrm{m} / \mathrm{z} 362$, B4) detected in 16/20 urine samples seemed to be a choice urinary marker as it appeared to have a higher \% peak area ratio when compared to ester hydrolysis plus dehydrogenation metabolite (B20). However, this metabolite was only detected in trace amounts in both the $C$. elegans and HLM models. Hence, structural elucidation could not be confirmed unless a reference standard is made available. Despite the relatively high $\%$ peak area ratio and detection in 16/20 urine samples, ester hydrolysis followed by monohydroxylation at the tert-leucine moiety $(\mathrm{m} / \mathrm{z} 366$, B 8$)$ metabolite was not reported among the 17 urine samples from the forensic psychiatric ward and prison in Haschimi et al.'s study [30]. Hence, the use of this metabolite as the urinary marker was not selected.

Finally, a comparison of the results obtained from incubations of 4F-MDMB-BINACA with three different models showed that all of them were in agreement with the tentatively identified urinary metabolites. C. elegans and HLM models detected all of the in-vivo metabolites (100\%), whilst HepG2 cells detected 7 out of the 8 in-vivo metabolites (87.5\%).
HepG2 model detected the major ester hydrolysis metabolite of 4F-MDMB-BINACA in abundance but the rest of the metabolites were found in a small amount. This might be due to the low activity of numerous metabolizing enzymes resulting in lower drug biotransformation [42]. Comparison of $C$. elegans with in-vivo metabolites showed that while C. elegans has limitations such as a low abundance of ester hydrolysis metabolite, in general, the metabolic profiles were in good agreement. The findings also supported that $C$. elegans possessed enzymatic activity that mimics the human metabolism of 4F-MDMB-BINACA. HLM possessed both the CYP and carboxylesterase enzymes accounted for biotransformation that matches with the in-vivo findings. It should be noted that the chemical structures of the tentative elucidated metabolites have not been unequivocally confirmed. With C. elegans model, scaling up of metabolites production for NMR spectroscopy could provide unequivocal structural elucidation of the metabolites. C. elegans has also been reported to form and trap active metabolites using trapping agents such as glutathione [28]. Scaling up these potential active metabolites conjugates allows the identification of potential metabolite toxicities thus giving an enhanced understanding of the toxicity mechanism of 4F-MDMB-BINACA. Therefore, C. elegans is a useful complementary metabolic model for SCBs, especially when a larger quantity of metabolites is required.

\section{Conclusions}

The present study showed that HLM proved to be a suitable predictive in-vitro - in-vivo extrapolation model for 4F-MDMB-BINACA. C. elegans demonstrated the ability to form all of the in-vivo metabolites and has the potential to be used as a complementary model to predict and characterize human metabolites, as well as identifying possible drug toxicities for emerging SCBs. For the identification of 4F-MDMBBINACA intake, ester hydrolysis (B22) and ester hydrolysis followed by dehydrogenation (B20) metabolites were identified as specific and relevant urinary markers. The difficulty in unequivocally identifying the structures of dehydrogenation metabolites including B20 by mass spectrometry demonstrated the need to utilise NMR spectroscopy. Future work will include isolation and confirmation of scaled-up reactions by C. elegans model to confirm the proposed chemical structure assignments to the metabolites identified here.

Acknowledgements The authors would like to thank Mr. Matin Simone for technical support in culturing HepG2 liver cells; and Ms. Sophie Winiarski and Ms. Jane Cameron for the guidance and technical support in the C. elegans experiments. This work was supported by the Australian Government Research Training Program Scholarship (to H.S.L.) and the Australian Research Council Discovery grant (to P.K.W.). 


\section{Compliance with ethical standards}

Conflict of interest The authors do not have financial or other relations that could lead to a conflict of interest.

Ethical approval This article does not contain any studies with human participants or animals performed by any of the authors.

Open Access This article is licensed under a Creative Commons Attribution 4.0 International License, which permits use, sharing, adaptation, distribution and reproduction in any medium or format, as long as you give appropriate credit to the original author(s) and the source, provide a link to the Creative Commons licence, and indicate if changes were made. The images or other third party material in this article are included in the article's Creative Commons licence, unless indicated otherwise in a credit line to the material. If material is not included in the article's Creative Commons licence and your intended use is not permitted by statutory regulation or exceeds the permitted use, you will need to obtain permission directly from the copyright holder. To view a copy of this licence, visit http://creativecommons.org/licenses/by/4.0/.

\section{References}

1. Cohen K, Weinstein AM (2018) Synthetic and non-synthetic cannabinoid drugs and their adverse effects - a review from public health prospective. Front Public Health 6:162. https://doi. org/10.3389/fpubh.2018.00162 (open access article)

2. Brandt S, Poovendran D (2019) 4F-MDMB-BINACA. Critical review report. World Health Organization Expert Committee on Drug Dependence (ECCD) Forty-second Meeting, pp 1-24. Geneva, 21-25

3. Banister SD, Longworth M, Kevin R, Sachdev S, Santiago M, Stuart J, Mack JBC, Glass M, McGregor IS, Connor M, Kassiou M (2016) Pharmacology of valinate and tert-leucinate synthetic cannabinoids 5F-AMBICA, 5F-AMB, 5F-ADB, AMB-FUBINACA, MDMB-FUBINACA, MDMB-CHMICA, and their analogues. ACS Chem Neurosci 7:1241-1254. https ://doi.org/10.1021/acschemneuro.6b00137

4. Schoeder CT, Hess C, Madea B, Meiler J, Müller CE (2018) Pharmacological evaluation of new constituents of "Spice": synthetic cannabinoids based on indole, indazole, benzimidazole and carbazole scaffolds. Forensic Toxicol 36:385-403. https:// doi.org/10.1007/s11419-018-0415-z (open access article)

5. Krotulski AJ, Mohr ALA, Kacinko SL, Fogarty MF, Shuda SA, Diamond FX, Kinney WA, Menendez MJ, Logan BK (2019) 4F-MDMB-BINACA: a new synthetic cannabinoid widely implicated in forensic casework. J Forensic Sci 64:1451-1461. https://doi.org/10.1111/1556-4029.14101

6. Krotulski AJ, Mohr ALA, Logan BK (2018) Trend report: Q4 2018. Synthetic cannabinoids in the United States. https://www. forensicscienceeducation.org/wp-content/uploads/2019/02/ Synthetic-Cannabinoid-Trend-Report_Detailed_2018-Q4.pdf. Accessed 1 Aug 2020

7. Krotulski AJ, Mohr ALA, Logan BK (2019) Trend report: Q1 2019. Synthetic cannabinoids in the United States. https://www. forensicscienceeducation.org/wp-content/uploads/2019/04/ Synthetic-Cannabinoid-Trend-Report_Detailed_2019-Q1.pdf. Accessed 1 Aug 2020

8. Krotulski AJ, Mohr ALA, Logan BK (2019) Trend report: Q2 2019. Synthetic cannabinoids in the United States. https://www. npsdiscovery.org/wp-content/uploads/2019/07/Synthetic-Canna binoid-Trend-Report_Detailed_2019-Q2.pdf. Accessed 1 Aug 2020
9. Krotulski AJ, Mohr ALA, Logan BK (2019) Trend report: Q3 2019. Synthetic cannabinoids in the United States. https://www. npsdiscovery.org/wp-content/uploads/2019/11/Synthetic-Canna binoid-Trend-Report_Detailed_2019-Q3.pdf. Accessed 1 Aug 2020

10. Fantegrossi WE, Moran JH, Radominska-Pandya A, Prather PL (2014) Distinct pharmacology and metabolism of K2 synthetic cannabinoids compared to $\Delta^{9}$-THC: mechanism underlying greater toxicity? Life Sci 97:45-54. https://doi.org/10.1016/j. lfs.2013.09.017 (open access article)

11. Tai S, Fantegrossi WE (2017) Pharmacological and toxicological effects of synthetic cannabinoids and their metabolites. In: Baumann MH, Glennon RA, Wiley JL (eds) Neuropharmacology of new psychoactive substances (NPS): the science behind the headlines. Springer International Publishing, Cham, pp 249-262

12. Richter LHJ, Flockerzi V, Maurer HH, Meyer MR (2017) Pooled human liver preparations, HepaRG, or HepG2 cell lines for metabolism studies of new psychoactive substances? A study using MDMA, MDBD, butylone, MDPPP, MDPV, MDPB, 5-MAPB, and 5-API as examples. J Pharm Biomed Anal 143:32-42. https ://doi.org/10.1016/j.jpba.2017.05.028

13. Li J, Liu C, Li T, Hua Z (2018) UPLC-HR-MS/MS-based determination study on the metabolism of four synthetic cannabinoids, ADB-FUBICA, AB-FUBICA, AB-BICA and ADB-BICA, by human liver microsomes. Biomed Chromatogr 32:e4113. https:// doi.org/10.1002/bmc.4113 (open access article)

14. Staeheli SN, Poetzsch M, Veloso VP, Bovens M, Bissig C, Steuer AE, Kraemer T (2018) In vitro metabolism of the synthetic cannabinoids CUMYL-PINACA, 5F-CUMYL-PINACA, CUMYL-4CN-BINACA, 5F-CUMYL-PINACA, CUMYL-4CNBINACA, 5F-CUMYL-P7AICA and CUMYL-4CN-B7AICA. Drug Test Anal 10:148-157. https://doi.org/10.1002/dta.2298

15. Yeter O, Öztürk YE (2019) Metabolic profiling of synthetic cannabinoid 5F-ADB by human liver microsome incubations and urine samples using high-resolution mass spectrometry. Drug Test Anal 11:847-858. https://doi.org/10.1002/dta.2566

16. Mogler L, Franz F, Rentsch D, Angerer V, Weinfurtner G, Longworth M, Banister SD, Kassiou M, Moosmann B, Auwärter V (2018) Detection of the recently emerged synthetic cannabinoid 5F-MDMB-PICA in 'legal high' products and human urine samples. Drug Test Anal 10:196-205. https://doi.org/10.1002/ dta.2201(openaccessarticle)

17. Westerink WMA, Schoonen WGEJ (2007) Cytochrome P450 enzyme levels in HepG2 cells and cryopreserved primary human hepatocytes and their induction in HepG2 cells. Toxicol In Vitro 21:1581-1591. https://doi.org/10.1016/j.tiv.2007.05.014

18. Guo L, Dial S, Shi L, Branham W, Liu J, Fang J-L, Green B, Deng H, Kaput J, Ning B (2011) Similarities and differences in the expression of drug-metabolizing enzymes between human hepatic cell lines and primary human hepatocytes. Drug Metab Dispos 39:528-538. https://doi.org/10.1124/dmd.110.035873

19. O'Brien PJ, Edvardsson A (2017) Validation of a multiparametric, high-content-screening assay for predictive/investigative cytotoxicity: evidence from technology transfer studies and literature review. Chem Res Toxicol 30:804-829. https://doi.org/10.1021/ acs.chemrestox.6b00403

20. Asha S, Vidyavathi M (2009) Cunninghamella - a microbial model for drug metabolism studies - a review. Biotechnol Adv 27:16-29. https://doi.org/10.1016/j.biotechadv.2008.07.005

21. Grafinger KE, Stahl K, Wilke A, König S, Weinmann W (2018) In vitro phase I metabolism of three phenethylamines 25D-NBOMe, 25E-NBOMe and 25N-NBOMe using microsomal and microbial models. Drug Test Anal 10:1607-1626. https://doi. org/10.1002/dta.2446 
22. Grafinger KE, Wilke A, König S, Weinmann W (2019) Investigating the ability of the microbial model Cunninghamella elegans for the metabolism of synthetic tryptamines. Drug Test Anal 11:721-729. https://doi.org/10.1002/dta.2544

23. Watanabe S, Kuzhiumparambil U, Winiarski Z, Fu S (2016) Biotransformation of synthetic cannabinoids JWH-018, JWH-073 and AM2201 by Cunninghamella elegans. Forensic Sci Int 261:33-42. https://doi.org/10.1016/j.forsciint.2015.12.023

24. Watanabe S, Kuzhiumparambil U, Nguyen M, Cameron J, Fu S (2017) Metabolic profile of synthetic cannabinoids 5F-PB-22, PB-22, XLR-11 and UR-144 by Cunninghamella elegans. AAPS J 19:1148-1162. https://doi.org/10.1208/s12248-017-0078-4

25. Watanabe S, Kuzhiumparambil U, Fu S (2018) In vitro metabolism of synthetic cannabinoid AM1220 by human liver microsomes and Cunninghamella elegans using liquid chromatography coupled with high resolution mass spectrometry. Forensic Toxicol 36:435-446. https://doi.org/10.1007/s11419-018-0424-y(opena ccessarticle)

26. Gaunitz F, Dahm P, Mogler L, Thomas A, Thevis M, Mercer-Chalmers-Bender K (2019) In vitro metabolic profiling of synthetic cannabinoids by pooled human liver microsomes, cytochrome P450 isoenzymes, and Cunninghamella elegans and their detection in urine samples. Anal Bioanal Chem 411:3561-3579. https ://doi.org/10.1007/s00216-019-01837-8

27. Zhang D, Yang Y, Leakey JEA, Cerniglia CE (1996) Phase I and phase II enzymes produced by Cunninghamella elegans for the metabolism of xenobiotics. FEMS Microbiol Lett 138:221-226. https://doi.org/10.1111/j.1574-6968.1996.tb08161.x (open access article)

28. Rydevik A, Hansson A, Hellqvist A, Bondesson U, Hedeland M (2015) A novel trapping system for the detection of reactive drug metabolites using the fungus Cunninghamella elegans and high resolution mass spectrometry. Drug Test Anal 7:626-633. https ://doi.org/10.1002/dta.1714

29. Wagmann L, Frankenfeld F, Park YM, Herrmann J, Fischmann S, Westphal F, Müller R, Flockerzi V, Meyer MR (2020) How to study the metabolism of new psychoactive substances for the purpose of toxicological screenings - a follow-up study comparing pooled human liver S9, HepaRG cells, and zebrafish larvae. Front Chem 8:539. https://doi.org/10.3389/fchem.2020.00539 (openaccessarticle)

30. Haschimi B, Mogler L, Halter S, Giorgetti A, Schwarze B, Westphal F, Fischmann S, Auwärter V (2019) Detection of the recently emerged synthetic cannabinoid 4F-MDMB-BINACA in "legal high" products and human urine specimens. Drug Test Anal 11:1377-1386. https://doi.org/10.1002/dta.2666

31. Richter LHJ, Beck A, Flockerzi V, Maurer HH, Meyer MR (2019) Cytotoxicity of new psychoactive substances and other drugs of abuse studied in human HepG2 cells using an adopted high content screening assay. Toxicol Lett 301:79-89. https://doi. org/10.1016/j.toxlet.2018.11.007

32. Choudhary MI, Khan NT, Musharraf SG, Anjum S, Atta-ur-Rahman (2007) Biotransformation of adrenosterone by filamentous fungus, Cunninghamella elegans. Steroids 72:923-929. https:// doi.org/10.1016/j.steroids.2007.08.002

33. Rydevik A, Bondesson U, Thevis M, Hedeland M (2013) Mass spectrometric characterization of glucuronides formed by a new concept, combining Cunninghamella elegans with TEMPO. J Pharm Biomed Anal 84:278-284. https://doi.org/10.1016/j. jpba.2013.06.012

34. Diao X, Huestis MA (2019) New synthetic cannabinoids metabolism and strategies to best identify optimal marker metabolites. Front Chem 7:109. https://doi.org/10.3389/fchem.2019.00109 (openaccessarticle)

35. Wang D, Zou L, Jin Q, Hou J, Ge G, Yang L (2018) Human carboxylesterases: a comprehensive review. Acta Pharm Sin B 8:699. https://doi.org/10.1016/j.apsb.2018.05.005 (open access article)

36. Thomsen R, Nielsen LM, Holm NB, Rasmussen HB, Linnet K (2015) Synthetic cannabimimetic agents metabolized by carboxylesterases. Drug Test Anal 7:565-576. https://doi.org/10.1002/ dta. 1731

37. Ross MK, Borazjani A, Wang R, Crow JA, Xie S (2012) Examination of the carboxylesterase phenotype in human liver. Arch Biochem Biophys 522:44-56. https://doi.org/10.1016/j. abb.2012.04.010 (open access article)

38. Di L (2019) The impact of carboxylesterases in drug metabolism and pharmacokinetics. Curr Drug Metab 20:91-102. https://doi. org/10.2174/1389200219666180821094502 (open access article)

39. Yeter EO, Yeter O (2020) In vitro phase I metabolism of the recently emerged synthetic MDMB-4en-PINACA and its detection in human urine samples. J Anal Toxicol. https://doi. org/10.1093/jat/bkaa017

40. Watanabe S, Vikingsson S, Åstrand A, Gréen H, Kronstrand R (2019) Biotransformation of the new synthetic cannabinoid with an alkene, MDMB-4en-PINACA, by human hepatocytes, human liver microsomes, and human urine and blood. AAPS J 22:13. https://doi.org/10.1208/s12248-019-0381-3

41. Wohlfarth A, Castaneto MS, Zhu M, Pang S, Scheidweiler KB, Kronstrand R, Huestis MA (2015) Pentylindole/pentylindazole synthetic cannabinoids and their 5-fluoro analogs produce different primary metabolites: metabolite profiling for AB-PINACA and 5F-AB-PINACA. AAPS J 17:660-677. https://doi.org/10.1208/ s12248-015-9721-0

42. Yokoyama Y, Sasaki Y, Terasaki N, Kawataki T, Takekawa K, Iwase Y, Shimizu T, Sanoh S, Ohta S (2018) Comparison of drug metabolism and its related hepatotoxic effects in HepaRG, cryopreserved human hepatocytes, and HepG2 cell cultures. Biol Pharm Bull 41:722-732. https://doi.org/10.1248/bpb.b17-00913 (open access article)

Publisher's Note Springer Nature remains neutral with regard to jurisdictional claims in published maps and institutional affiliations. 